\title{
La experiencia estudiantil universitaria en América Latina: una revisión de literatura
}

The University Student Experience in Latin America: a Literature Review

A experiência do estudante universitário na América Latina: uma revisão da literatura

Diego Mauricio Barragán-Díaz* (iD hetps://orcid.org/0000-0003-4196-3543

\section{Doi: 10.17227/rce.num78-6708}

Para citar este artículo: Barragán Díaz, D. (2020). La experiencia estudiantil universitaria en América Latina: una revisión de literatura. Revista Colombiana de Educación, 78, 147-171. https://doi.org/10.17227/rce. num78-6708

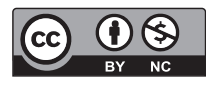




\section{Resumen}

Este artículo de revisión analiza alternativas de indagación, cercanas a la sociología, utilizadas para comprender la experiencia estudiantil en la universidad. Se revisó literatura producida en América Latina y complementada con algunos textos escritos en Europa y Estados Unidos. Ante la diversidad de los abordajes y la variedad de los textos, se planeó la estructura del artículo así: se inicia con un análisis de obras que se toman como referentes teóricos y metodológicos, luego, se abordan textos que tratan de las actividades que desarrollan los estudiantes, los espacios que construyen o donde participan y los vínculos que generan en su paso por la universidad. Son recurrentes las investigaciones en las que se toman dimensiones vinculadas al proceso educativo y se dejan de lado las relaciones de los estudiantes con grupos, hechos, situaciones problemas que son frecuentes en la cotidianidad de sus países. En estos contextos, las personas toman rutas alternas para desarrollar su proceso de formación, buscando recursos que les permitan continuar y herramientas para entender o modificar su condición individual en entornos sociales conflictivos. En esta lucha, las personas sufren una serie de transformaciones que se evidencian en la experiencia estudiantil.

\section{Palabras clave}

experiencia estudiantil; universidad; adultos jóvenes; sociología de la educación

\section{Keywords}

student experience; university; young adults; sociology of education

\begin{abstract}
This review article analyzes alternatives of inquiry, close to sociology, used to understand the student experience at the university. The authors reviewed literature produced in Latin America and supplemented with some texts published in Europe and the United States. Given the diversity of the approaches and the variety of texts, the structure of the article is as follows: it begins with an analysis of works that are taken as theoretical and methodological references, then the authors address texts dealing with the activities developed by the students, the spaces they build or where they participate, and the links they generate as they go through the university. In numerous investigations on student experience, the authors deal with dimensions linked to the educational process and leave aside the students' relationships with groups, facts, situations or problems which are frequent in their countries. In these contexts, people take alternate routes to develop their training process, seeking resources that allow them to continue, as well as tools to understand, or modify, their individual condition in conflictive social environments. In this struggle, people undergo a series of transformations that are evident in the student experience.
\end{abstract}

\begin{abstract}
Resumo
Este artigo de revisão analisa alternativas de pesquisa, próximas à sociologia, usadas para entender a experiência do aluno na universidade. Revisamos a literatura produzida na América Latina e foi complementada com alguns textos escritos na Europa e nos Estados Unidos. Dada a diversidade de abordagens e a variedade de textos, a estrutura do artigo foi planejada da seguinte forma: começa com uma análise de trabalhos que são tomados como referenciais teóricos e metodológicos e, em seguida, textos que tratam das atividades realizadas pelo estudantes, os espaços que constroem ou onde participam e os vínculos que geram em seu tempo na universidade são tratados. São recorrentes as pesquisas em que dimensões relacionadas ao processo educacional são tomadas e são deixadas de lado as relações dos alunos com grupos, fatos, situações ou problemas que são frequentes no cotidiano de seus países. Nesses contextos, as pessoas seguem caminhos alternativos para desenvolver seu processo de formação, procurando recursos que Ihes permitam continuar e ferramentas para entender ou modificar sua condição individual em ambientes sociais conflitantes. Nesta luta, as pessoas sofrem uma série de transformações evidentes na experiência do aluno.
\end{abstract}

\section{Palavras-chave}

experiência do estudante universidade; adultos jovens; sociologia da educação 


\section{Introducción}

Las perspectivas sociológicas para hablar de los estudiantes universitarios son múltiples e implican profundizar en un campo amplio y complejo (Dubet, 2010; Gómez y Alzate, 2010). Una tendencia usual es establecer límites entre los roles de estudiante y de joven, separación que resulta inconveniente para entender a jóvenes que participan en educación (Dubet, 2005). En efecto, los jóvenes habitan un mundo lejano del de los adultos; en consecuencia, una forma de entenderlos es enfocarse en su participación en grupos (políticos, sociales, deportivos, juveniles, etc.), en consumos (desde música hasta drogas) o en cualquier dimensión que muestre sus singularidades (Pinzón, Garay y Suárez, 2008). Algunas investigaciones presentan a los estudiantes como extraños, atípicos; como contraventores de actividades institucionales, de un orden social o de la imagen ideal de "buen estudiante". A pesar de esto, la distancia con los jóvenes, la complejidad de sus relaciones y la particularidad de sus actuaciones son problemas constantes, que necesitan desarrollar marcos que permitan encontrar miradas alternativas.

Buscando caminos para sintetizar las investigaciones sobre el tránsito de los jóvenes por las universidades latinoamericanas, se formula una pregunta: ¿Qué alternativa puede agrupar las diferencias y las complejidades de las interpretaciones sobre los estudiantes? En la búsqueda de una respuesta, se encontró la experiencia estudiantil (Arango, 2006; Carli, 2012; Dubet, 2010; Guzmán, 2002). Hoy, se tiene un acumulado significativo de investigaciones sobre la experiencia estudiantil, o fenómenos cercanos, en las que la formación tiene un rol importante, pero se abren dimensiones sociales, económicas o momentos concretos que inciden en el paso de los jóvenes por la universidad. Es decir, en sus experiencias han quedado marcadas las transformaciones de los individuos, su formación, su vinculación a comunidades o dinámicas de sectores del conocimiento, y los cambios abruptos que se han presentado en la sociedad inmediata que afectan su experiencia universitaria.

Frente a la complejidad del tema, y después de una investigación empírica, Dubet (2010) plantea la categoría Experiencia como una alternativa para vincular a los individuos que participan en los procesos sociales. De tal manera, la experiencia individual forma parte de una experiencia social. Durante su proceso educativo, el individuo, como protagonista, incorpora experiencias que le permiten ver, creer, sentir y actuar frente a situaciones concretas o en las relaciones con otras personas. Los individuos participan en interdependencias con otros (Elías, 1990), son actores de reparto o protagonistas de un proceso educativo. Allí, aprenden a desenvolverse identificando alternativas en su formación universitaria y en sus intercambios cotidianos. 
Los estudiantes se desenvuelven en una sociedad en particular que tiene unas lógicas (Bourdieu, 2004) y que establece las condiciones para su desarrollo. La experiencia educativa es situada y tiene un tiempo (Carli, 2012), es de carácter social y obedece a estructuras cognitivas, a dinámicas de formación y a comportamientos establecidos (Lahire, 2006). Se habla de que las configuraciones de las experiencias estudiantiles dependen de las particularidades sociales y económicas de la sociedad donde se adelantan.

En el artículo se realiza una revisión de las alternativas utilizadas, cercanas a la sociología, para el análisis de la experiencia estudiantil en la universidad. A pesar de corresponder a lugares y momentos concretos, se trató de analizar los rasgos permanentes y vinculados a la experiencia estudiantil. Se parte de que las investigaciones tienen diversos enfoques metodológicos y teóricos, que presentan un panorama variado y complejo para comprender la experiencia estudiantil (Gómez y Alzate, 2010). La alternativa elegida se apoya en Dubet (2005), centrada en estudios de tipo cualitativo en los cuales los estudiantes son los protagonistas, narran sus experiencias, identifican hitos en su formación y establecen vínculos con la sociedad inmediata.

Dado que es una investigación documental, las fuentes son artículos de revistas científicas, tesis doctorales, capítulos o libros, resultado de investigaciones cualitativas, cercanas a la sociología de la educación, realizadas en América Latina y complementadas con algunos estudios sobre Europa y Estados Unidos. Las partes del artículo corresponden a la organización de las fuentes consultadas: la primera menciona las obras consideradas como referentes básicas, son los pilares sobre los cuales se apoya la investigación acerca del tema, y combinan textos producidos en América Latina y Europa. La segunda parte aborda las actividades principales que desarrollan los estudiantes universitarios en la región: estudiar, trabajar y militar. La tercera, se refiere a los espacios, generalmente extraescolares, donde transcurre la vida universitaria, que se convierten en significativos para los estudiantes. La cuarta, indaga sobre los vínculos con comunidades académicas, intercambios (locales, nacionales e internacionales) y grupos diversos donde participan los estudiantes durante su formación. Al estudiar los referentes básicos, las actividades, los espacios y los vínculos que se generan en el proceso de formación, se logra una aproximación a las investigaciones de la experiencia estudiantil en América Latina.

\section{Los referentes básicos}

Los referentes básicos son investigaciones que parten, específicamente, de la indagación sobre la experiencia estudiantil universitaria, salvo Rockwell (1997) que hace su trabajo en la escuela. En los distintos estudios se 
observan las condiciones y las dinámicas de la educación superior actual, que ha sufrido transformaciones por la masificación, las reformas y la participación de los capitales privados. Sin embargo, los estudiantes ya no forman parte de las modelizaciones pasadas, por lo cual parecen unos desconocidos para quienes se encargan de la orientación de las instituciones. Entre las investigaciones que han abordado el problema en distintos lugares y momentos podemos mencionar las de Rockwell (1997), Dubet (2005), French (1997), Yair (2008) y Carli (2012).

Rockwell, ante las reiteradas interpretaciones administrativas, legalistas y deterministas en la educación, establece una alternativa: la experiencia escolar. Esta varía de una sociedad a otra, de una escuela a otra; es una experiencia situada (Rockwell, 1997). Allí convergen diversas facetas que se yuxtaponen durante los cinco años que están los niños en ella: dinámicas históricas, orientaciones burocráticas, decisiones políticas, variaciones locales, interpretaciones de maestros y estudiantes, todas influyen en la experiencia. Esta autora destaca la realidad cotidiana y las prácticas como producto del contexto formativo real tanto de maestros como de estudiantes. A partir de prácticas se constituye la experiencia, producto de quienes participan en el proceso educativo. La escuela se presenta desde una contradicción: por un lado, reproduce formas de organizar el trabajo, las pautas de diferenciación social y relaciones de poder de la sociedad mayor; y por el otro, es transmisora de valores y prácticas superiores a las prevalecientes en la realidad circundante, formando ciudadanos para el futuro, para una sociedad mejor (Rockwell, 1997).

Dubet realiza con los estudiantes ${ }^{1}$ una investigación sobre los estudiantes universitarios que destaca rasgos del sistema educativo francés, la masificación, la diversificación de la oferta, y su relación con las distintas características y problemas que afrontaban los jóvenes que entraban a la universidad. A pesar de establecer relaciones entre las dos dimensiones, la base era cómo se vinculaban los jóvenes y los estudiantes en unas condiciones sociales y educativas complejas (Dubet, 2005). Así, establece que los individuos permanecen durante un tiempo considerable en instituciones educativas, por lo cual este periodo tiene repercusiones en su vida social.

1 Dentro de las obras destacadas de sociología de la experiencia estudiantil se encuentra la de Dubet y Martucelli, En la escuela, sociología de la experiencia escolar, publicada en español en 1998. Ellos proponen una corriente para la sociología de la educación cuya base es la investigación empírica, en la cual el individuo se adapta a los procesos de socialización educativos; estas relaciones constituyen las experiencias escolares. Esta investigación se realizó durante tres años en escuelas primarias, básicas y secundarias y se trabajó con estudiantes, padres y maestros. Si bien la obra de Dubet y Martucelli es fundamental para la sociología de la experiencia, en este artículo se opta por su texto Los estudiantes, por su relación directa con la temática y la población objeto de estudio. 
Este autor sostiene que en la actualidad las personas y sus condiciones de vida han cambiado significativamente, incluso las dinámicas y las relaciones entre estudiantes y maestros son diferentes. Las miradas de los estudiantes están inmersas en un remolino creciente de complejidad. Señala Dubet (2005) que a pesar de los cambios de referentes en la juventud, se destaca su paso por la universidad: es un espacio que transforma sus vidas; pese a las dificultades actuales, ofrece posibilidades de futuro. Los años que comparten en el proceso de formación modifican sus subjetividades, aunque guarden niveles altos de autonomía, establecen lazos, criterios, participan y se convierten en parte de algo.

En 1997, French publicó una investigación sobre las experiencias de ocho estudiantes universitarios, en la cual se evidenciaron cambios en su proceso de formación, desde que ingresaron hasta que terminaron; la observación inició en 1989 y finalizó en 1993. El autor aclara que no observó aspectos formales relacionados con los procesos académicos, sino que se aventuró en los aspectos que tienen que ver con su vida privada y su mundo. Desde luego, se establecieron diálogos con su formación, pero las transformaciones se daban en otros aspectos. En el proceso, se identificaron tres transformaciones: la exposición social, participar en nuevas redes de personas y formar parte de ellas; la exposición académica, su contacto con el saber, con discursos que ofrecen los compañeros, los profesores, la universidad y los vincula a sus representaciones del mundo; por último, la conciencia de sí, su ubicación en las relaciones y los espacios sociales, un alejamiento de la dependencia con otros (familia y amigos) en correspondencia con el avance del proceso de formación. De tal modo, el paso por la universidad los expuso a nuevas y diferentes realidades, y les ofreció a los estudiantes maneras de ver, actuar y enfrentar el mundo. La educación los transformó.

Una visión distinta de la experiencia la plantea Yair (2008), quien enfoca su estudio en individuos, a quienes invitó a identificar claves (keys) que consideran positivas y contribuyeron a su autodescubrimiento durante la experiencia universitaria. Ubicó tres dimensiones, cercanas a French (1997): la primera, se inscribe en la relación con el conocimiento, la vinculación a formas de interpretar la realidad; la segunda, en la relación de las personas como miembros de una comunidad con una dinámica propia; la tercera, se refiere al autorreconocimiento del individuo (Yair, 2008). Los participantes identificaron que episodios cortos o intensos se convirtieron en permanentes y transformaron su vida (Yair, 2008). Incluso, los hechos claves que se relataban fueron calificados como extraordinarios. El resultado de la investigación revela que las claves de la experiencia universitaria ayudan a los participantes a construir la personalidad, la identidad y el autoconcepto (Yair, 2008). 
Otra forma de definir la experiencia es la propuesta de Carli (2012). Su investigación indagaba sobre las experiencias que tuvieron los estudiantes en el proceso educativo, estableciendo sus conexiones con el periodo de crisis social, económica y política de la Argentina en 2001. El interés surgió de los cambios y las dificultades en el sistema educativo, a la par que las distancias entre profesores y estudiantes crecían; en general, un desconocimiento de las personas que adelantaban su proceso de formación en una época y un contexto complejos. Indagar a los protagonistas ofrece una mirada distinta del proceso de formación, la situación y las vivencias de los jóvenes y, sobre todo, alternativas para interpretar la crisis de 2001 y sus repercusiones en los años siguientes.

A pesar de la crisis económica y social, la formación establece las relaciones entre la socialización y los individuos, entre lo que se transmite y lo que los jóvenes asimilan (Carli, 2012). El tránsito por la universidad en periodos de crisis, pese a los problemas estructurales, ofrece a los estudiantes espacios de realización, donde adoptan, por un lado, parámetros cognitivos que les dan herramientas para entender sus realidades e incursionar en alternativas, y, por otro, parámetros morales que les brindan pautas de comportamiento; su pertenencia a grupos dentro de la universidad les ofrece un espacio de construcción y discusión colectiva que no encuentran en otros lugares.

Aunque se realizaron en lugares distintos (México, Francia, Inglaterra, Israel y Argentina), los estudios sobre la experiencia estudiantil tienen puntos en común. Se presentan situaciones poco alentadoras en los contextos sociales y económicos, especialmente, en el ámbito educativo, con un ingrediente adicional: la pérdida del valor social de la educación (Rojas, 2009). En consecuencia, los estudiantes se encuentran en un proceso de formación que requiere esfuerzo, recursos, entrega y observan en su cotidianidad conflictos por derechos fundamentales, como salud, trabajo y educación, que les muestran escenarios donde las personas pierden sus derechos y el futuro parece poco esperanzador. El estado de caos social sumado a su propio contexto son determinantes que influyen en la experiencia de los estudiantes. Vincularlos con grupos en la universidad ofrece espacios para tejer redes con personas vinculadas a los procesos de formación; son relaciones sociales que se forman desde la universidad. El individuo presenta cambios en su comportamiento durante el proceso de formación, se asimilan parámetros morales que forman parte de grupos o de segmentos de la institución, y se establecen cánones de comportamiento de las personas. Según los acercamientos, durante su proceso de formación en la universidad, los estudiantes cambian su vida y esto se evidencia en sus experiencias. 


\section{Las actividades destacadas}

Tradicionalmente, se acepta la hipótesis de que la actividad central de los estudiantes universitarios es estudiar. Esto implica observarlos desde su relación con su formación, suponiendo que solo se dedican a sus deberes académicos. Desde hace un tiempo, el estudio se combina con actividades que, algunas veces, requieren una cantidad de tiempo y esfuerzo superior a los empleados en el aprendizaje. Actualmente, los estudiantes participan en actividades deportivas, en algunos casos deben conseguir un empleo, se vinculan a redes sociales o grupos con intereses no académicos, buscan formas de entretenimiento que se distancien de su formación, exploran nuevos espacios que les brinden confianza y en los que no se sientan juzgados por sus capacidades intelectuales; de tal manera, han creado un nuevo concepto que implica mucho más que estudiar. Para el caso de América Latina, las investigaciones destacan tres actividades que han sido constantes: estudiar, trabajar y militar.

Es claro que estudiar es la actividad principal para un estudiante. Haciendo la analogía con los estudios del trabajo, ser estudiante universitario se toma como un "oficio" (Arango, 2006) en el que el "trabajador" pertenece a una institución, y asume compromisos y obligaciones relacionadas con el aprendizaje. Como los jóvenes llegan a identificar una situación, deben aprender y aplicar las reglas o normas que imperan en la institución. Son espacios sociales estables, acciones ordenadas en las instituciones y desarrolladas por los individuos. A continuación, se presentan algunas investigaciones acerca del estudiar; se parte de la adaptación al proceso y a nuevos escenarios (Benavides, et al., 2014; Malinowski, 2008; Roldán, 2014), cómo desempeña su rol de estudiante (Gómez y Alzate, 2010) y los conflictos que enfrenta; se destacan el fracaso (Arango, 2006; Graff, 1995; Kaplan, 2008; Nobile y Arroyo, 2015), la diversidad (Casillas, De Garay, Vergara y Puebla, 2001) y el contexto donde desarrollan su proceso (Guevara, 2009).

La vinculación de los estudiantes implica un ajuste en las expectativas de las personas y el cumplimiento con las normas y las dinámicas de la universidad. Algunas investigaciones que tienen como tema la deserción estudiantil examinan el primer año de vinculación a partir del problema de adaptación, por la importancia que el fenómeno ha tomado. En un estudio sobre la adaptación de los jóvenes a la universidad, Benavides et al. (2014) observaron las relaciones entre las expectativas y las vivencias de los jóvenes, en los primeros momentos de vinculación a la universidad. Encontraron que la relación depende de la autonomía y madurez de los estudiantes. La tensión entre expectativas y realidad los afecta emocional, cognitiva y motivacionalmente, y se relaciona con el apoyo institucional y la carrera que desarrollan. No es solo el individuo cuando se enfrenta 
a su nuevo proceso; sus experiencias se acumulan. Muchos encuentran que su proceso de formación no satisface sus expectativas, lo que se traduce en frustración.

Según Roldán (2014), la adaptación a la universidad durante el primer año es importante para aumentar la retención, ya que ayuda a los jóvenes a encontrar un espacio de posibilidades para sí, para sus interacciones, y a disminuir la deserción derivada de la incapacidad de permanecer en un contexto exigente y hostil. La experiencia en los jóvenes durante el primer año es trascendental (Roldán, 2014); en este tiempo se vinculan a la cultura y las dinámicas institucionales y el sujeto encuentra su lugar para desarrollar su experiencia estudiantil. Es un sujeto en un nuevo escenario, donde teje lazos con las personas y debe impregnarse de la lógica de la institución para continuar y transformar su vida.

Los estudiantes desde esta perspectiva se integran a procesos de formación intelectual y de inserción social dentro de una comunidad, y es su responsabilidad la forma como desarrolla su camino en el aprendizaje (Gómez y Alzate, 2010). Es necesario abandonar la concepción de los estudiantes como recipientes que se deben Ilenar de conocimientos; contrario a esto, se hace indispensable asignarles el protagonismo del proceso de aprendizaje donde se establecen vínculos con el conocimiento, las personas, la formación disciplinar y los momentos donde participan. Son dimensiones que las personas tejen durante su formación. La vinculación de los estudiantes no se limita a la matrícula en la universidad (Malinowski, 2008); su afiliación crea el vínculo con el estudio y se convierte en el centro de la experiencia estudiantil. Los estudiantes deben atravesar un proceso de adaptación individual a los códigos intelectuales, de comportamiento y de desempeño establecidos en la universidad (Guevara, 2009). Es su decisión conocer y cumplir las reglas de juego (Guevara, 2009).

Cuando el individuo no aprende las dinámicas institucionales, se generan múltiples conflictos, ${ }^{2}$ que afectan de manera directa su experiencia estudiantil (Graff, 1995). Muchos jóvenes llegan a la universidad con unas expectativas muy altas acerca de su formación y lo primero que encuentran es que sus profesores no Ilenan esas expectativas. Encuentran profesores sin formación y sin argumentos, que se alejan del ideal que ellos tenían, y en cambio asumen una posición pasiva frente a las experiencias y generan un choque entre las expectativas de los estudiantes y las razones y los recursos que les presentan. Kaplan (2008) sostiene que los prejuicios o las expectativas de los maestros inciden en el rendimiento de los estudiantes; señala la importancia de los juicios profesorales para moldear el desempeño y las trayectorias de estos (Nobile y Arroyo, 2015).

2 Los conflictos pueden ir desde el aburrimiento en clase hasta la deserción y el fracaso escolar. 
Desde hace algún tiempo, ha sido constante en muchos jóvenes se dediquen a estudiar y trabajar. Hipotéticamente, el tiempo que se invierte en el estudio retrasa su entrada al mundo adulto, en especial al laboral. Pero, en la actualidad esto se ha puesto en duda. La experiencia universitaria va de la mano con la laboral, en algunos casos en ocupaciones de segundo orden, en otros en actividades donde se pueden desempeñar profesionalmente; esto depende de la relación con el contexto económico y social. Paoloni (2011) indaga sobre dos opciones: o bien los jóvenes durante su periodo de formación inician su trayectoria profesional, o consiguen trabajos de subsistencia que les permitan terminar sus estudios. Los resultados se orientan a la segunda opción: los estudiantes consiguen empleos para obtener dinero y continuar con sus estudios, en especial a través de trabajos menores que no guardan relación con su formación profesional. Un pequeño grupo se desempeña en empleos relacionados con la carrera, pero sin vinculación laboral estable, la mayoría tiene o ha tenido contratos temporales. Entonces, los trabajos son precarios, con baja remuneración, por corto tiempo y sin relación con sus estudios (Paoloni, 2011).

Vázquez (2009) plantea que los estudiantes que trabajan deben emplear su tiempo y su fuerza en dos actividades demandantes; esto debería obligar a las instituciones a pensar alternativas a los planes de estudio que en los procesos de formación están diseñados para personas dedicadas de tiempo completo a la universidad. La indagación se centró en el perfil socioeconómico y el desempeño académico. Como resultado, Vázquez asegura que la condición laboral incide en la trayectoria académica de los estudiantes, disminuye su rendimiento, pero amplía sus posibilidades laborales en el futuro.

Ruesga, Silva y Monsueto (2014) analizaron cómo influye la experiencia laboral en el desempeño académico de los estudiantes. Concluyeron que el trabajo los afecta negativamente si ocupan quince o más horas a la semana, pero si ocupan menos horas hay un efecto positivo en el desempeño académico y las perspectivas laborales. La decisión de entrar al mercado de trabajo depende de la edad y del dinero que les aporte la familia (Ruesga et al., 2014).

En el mismo sentido, Weiss, Klein y Gravenhorst (2014) consideran que la transición entre la universidad y el trabajo siempre es un momento crítico de la integración de las personas al mundo productivo, ya que de alguna manera se trazan las rutas que seguirán en el mundo laboral a largo plazo. Desde luego, dependen en gran medida del contexto socioeconómico en que se estudia; en este caso, el estudio analizó el caso de Alemania, donde los egresados tienen opciones de vincularse rápidamente al mercado laboral, lo que difiere de lo mostrado por investigaciones realizadas en México (Vázquez, 2009) o en Colombia (Barragán, 2017). El mercado de trabajo y las experiencias de las personas cambian significativamente, 
incluso las posibles trayectorias laborales. Algo que puede ser cercano en el planteamiento de Weiss et al. (2014) es el hecho de que existen diferencias entre las personas que voluntariamente toman la decisión de trabajar en determinadas áreas o condiciones laborales y quienes no. La participación voluntaria permite asumir el trabajo de forma diferente. La pertenencia a redes, el contacto con ciertas personas, el desarrollo de sus actividades los impacta de forma positiva en su desempeño y en las relaciones futuras (Weiss et al., 2014).

Dentro de la universidad latinoamericana, otra actividad aparece como relevante cuando se caracteriza a los estudiantes: militar. Consiste en la participación voluntaria y activa en un proceso público para la toma de decisiones. Las crisis sociales y económicas, que de tanto en tanto se repiten en la región, hacen que algunos estudiantes tomen la militancia como una forma de manifestación. No se puede relacionar directamente con desigualdades sociales, los problemas económicos, los gobiernos de facto, las dictaduras u oligarquías que gobiernan en la región; cada época cambia la relación de los estudiantes con sus experiencias concretas.

Desde el hito de Córdoba en 1918 hasta la actualidad, algunas interpretaciones han girado alrededor de una participación política de los estudiantes. En Colombia, Acosta, Cubides y Galindo (2011) indagaron sobre las prácticas y los sentidos que estudiantes universitarios asignan al ámbito político, siguiendo la tradicional división entre universidades públicas y privadas. Fueron varios los temas abordados, entre ellos se destaca cómo se plantea la categoría de Jóvenes que desarrollan un proceso de formación que les permite tener horizontes diferentes a los que se encuentran al margen de la educación superior. Una conclusión es que la institución influye de forma directa en las orientaciones y representaciones políticas de los jóvenes.

Muchos imaginarios son preconcebidos desde los grupos primarios, familia y amigos, que entran a compaginar o rechazar la postura institucional. La influencia de las orientaciones institucionales sobre los jóvenes parte de dos dimensiones: la primera, corresponde a las orientaciones neoliberales de autofinanciación de la educación, responsabilidad individual, la institución como un espacio de moratoria social y las pocas condiciones de trabajo digno al egresar; la segunda se refiere a las instituciones que han hecho un esfuerzo en el proceso de disciplinamiento de los estudiantes, estableciendo pautas de comportamiento, rituales, rutinas, una dinámica que estos deben cumplir y que en algunos casos son más valorados que las actividades académicas (Acosta et al., 2011). Se establece así un vínculo en las representaciones y prácticas políticas de los estudiantes en correspondencia directa con las dinámicas y las orientaciones institucionales. 
También Murcia (2006) sostiene que los imaginarios de los estudiantes y docentes sobre la universidad en América Latina son resultado de un proceso histórico que inició con el Manifiesto de Córdoba en 1918, y se ha reforzado con hitos hasta hoy. Según Murcia (2006), estas imágenes viven y orientan las experiencias de los estudiantes, lo cual entra en relación con las dinámicas de las reformas que se establecieron en los noventa y transformaron las lógicas de la universidad afectando a todos sus actores. Según Camou, Prati y Varela (2014), la militancia siempre ha estado en la vida de los estudiantes universitarios en América Latina. Hoy, los jóvenes son diferentes e identifican tres tipos de participación política que son resultado de la experiencia estudiantil que teje vínculos entre trayectorias personales, lógicas de la institución (ámbitos de formación) y situaciones de la realidad social y política concreta (Camou et al., 2014).

Las transformaciones sociales afectan la participación política y desplazan la imagen ideal de estudiante comprometido con una agrupación y un partido; hoy, la participación de los jóvenes es diferente (Picotto y Vommaro, 2010). Las organizaciones tradicionales se asocian o se responsabilizan de hechos o conflictos intensos que propiciaron o no supieron resolver. Las agrupaciones independientes se constituyen en un espacio de participación de los jóvenes, como refugios y a partir del establecimiento de prácticas, actores, miradas y herramientas. La experiencia de los participantes es el medio para expresar el cambio en las organizaciones. Son formas situadas de lo político, no aspiran a programas ideológicamente trascendentes, sino que sirven de refugio para los jóvenes que necesitan un punto de referencia. Resulta interesante que ante el agotamiento de los términos políticos tradicionales, aparecen afectos y situaciones particulares que solidifican los lazos entre las personas que participan.

En países como Chile y Colombia, se destacan las reivindicaciones sobre una educación superior pública y de calidad (Ouviña, 2012). Es notable el malestar que causa a egresados universitarios contraer deudas con entidades de crédito públicas y privadas por cinco o más años para poder pagar sus estudios; independientemente de su vinculación a universidades públicas o privadas, los estudiantes salían con las mismas deudas. Este caso es una muestra de que la participación de los estudiantes como miembros de un movimiento estudiantil (Archila, 2012; Bonavena y Millán, 2012; Donoso, 2014) no siempre corresponde con las tradiciones de militancia estudiantil imperantes en la región. Estudios diversos (Amaya, Gómez y Otero, 2007; Carrasco, 2010; Cubides y Guerrero, 2012; Larotonda, 2007; Lozano, 2009; Lozano y Alvarado, 2011; Mira, 2011; Sandoval y Hatibovic, 2010) dan cuenta de que en las últimas décadas la militancia estudiantil ha variado según los hechos, personas o situaciones particulares; lo que Ileva a pensar que el nexo entre política y vida estudiantil no está predeterminado por las ideologías imperantes. 
Tomando uno de los múltiples caminos que se abrieron en la militancia estudiantil, Blanco (2014c) estudia las reivindicaciones y las demandas de movimientos feministas o de diversidad sexual en agrupaciones universitarias. Se observan las estrategias frente al género para la vinculación a movimientos, y la politización de las identidades sexuales en procesos colectivos. Los acontecimientos externos marcan a las personas y modifican las dinámicas internas, que se reflejan en la militancia en agrupaciones diferentes en la universidad. Es un nuevo territorio político. Son nuevas redes de socialización donde confluyen agrupaciones estudiantiles y experiencias personales; son alternativas en las que personas con orientaciones e intereses similares pueden participar sin necesidad de vincularse a la militancia tradicional, y que no corresponden a una imagen estereotipada.

A pesar de que estudiar es el centro de las actividades observadas en los estudiantes universitarios en América Latina, trabajar y militar ocupan lugares importantes en investigaciones sobre las experiencias estudiantiles. Las personas son distintas y las dinámicas de las tres actividades cambiaron. Estudiar no solo se presenta como el centro del ejercicio, sino como un proceso en el cual intervienen diferentes dimensiones que inciden en el aprendizaje y la formación de los estudiantes. Trabajar se propone como una alternativa para terminar sus estudios frente a las pocas opciones de bienestar que se ofrecen a los estudiantes de los países de la región; desde luego, esto implica destinar tiempo, esfuerzo y recursos a dos actividades, lo cual desplaza un poco el centro del proceso de aprendizaje. Militar se presenta como una opción de encontrar nuevos espacios para jóvenes que necesitan vínculos con grupos de personas que comparten sus inquietudes y sus demandas. Así, son múltiples las actividades en que intervienen los estudiantes en su experiencia estudiantil, pero estudiar, trabajar y militar son las que reciben mayor atención en la América Latina.

\section{Los espacios de la vida universitaria}

En la tradición de sociología de la educación, el espacio se toma como un lugar clave en la integración del individuo a la vida universitaria, su vinculación a las actividades académicas y su socialización como parte de una comunidad (Guzmán, 2002). Recientemente, ante los cambios en las prácticas y los hábitos de los jóvenes, los espacios universitarios se convirtieron en lugares para desarrollar su vida más allá de las actividades académicas. Esto abre un abanico de posibilidades para que los jóvenes participen y construyan sus espacios. Desde estos puntos de vista, se encontraron varias investigaciones. Aunque el interés del artículo son las experiencias en la universidad, se mencionan dos que analizan la escuela y el colegio (Duschatzky, 2008; Kessler, 2002), que se incluyen por su importancia argumentativa. Luego se aborda cómo se refleja o se vive la 
cotidianidad de los estudiantes en espacios de la universidad (Barnes, 1992; Blanco 2014a y 2014b; Chávez, 2008). Finalmente, se habla de territorios de los estudiantes (Díaz, 2012; Rodríguez, 2011; Soler, Vilá, Fullana, Planas y Pallisera, 2011) y de cómo las crisis del contexto social afectan a la universidad (Zibecchi 2002).

El texto de Kessler (2002) aborda la experiencia estudiantil estableciendo las relaciones entre socialización e individuo en un nuevo contexto. La escuela cambió significativamente con las transformaciones sociales que se han dado desde finales del siglo xx. Las construcciones conceptuales sobre la escuela distaban de su funcionamiento, las relaciones y los comportamientos de los estudiantes se diferenciaban significativamente de lo que se creía debían ser los estudiantes en la escuela. La realidad era distinta y los estudiantes distantes. No se tenían poblaciones homogéneas de estudiantes, sino grupos heterogéneos. Lo anterior, asociado a la falta de institucionalidad genera transformaciones en la socialización y en la subjetividad de los estudiantes; por ello sus referentes cambian y sus escalas de valoración también. Las particularidades de los individuos o los grupos toman más fuerza en los procesos educativos que las orientaciones homogéneas que caracterizaban la escuela, esta se deja permear por los discursos y las prácticas que imperan en el contexto social. El espacio escolar ahora es el lugar donde convergen los procesos de socialización, los aspectos imperantes en el medio social y las necesidades y las expectativas de los jóvenes (Kessler, 2002).

A propósito de secundaria, Duschatzky (2008) afirma que si bien la experiencia es desarrollada por un grupo de individuos, ellos encuentran estructuras establecidas en el colegio, dinámicas en las relaciones sociales y contenidos en el campo cultural estableciendo unas relaciones entre el individuo que adelanta su proceso de formación y las dinámicas que se desarrollan en su entorno, y que él debe manejar para incorporarse en el sistema y tener una trayectoria aceptable. Duschatzky (2008) centró sus análisis de las experiencias en los testimonios de los estudiantes y encontró que el ámbito institucional no tenía una influencia significativa en los jóvenes; asimismo, que frente al contexto social conflictivo y poco esperanzador donde habitaban, encontraban en la escuela un espacio de construcción y proyección de su futuro.

En cuanto a las investigaciones sobre los estudiantes universitarios, Barnes (1992), desde una perspectiva antropológica, intenta establecer los vínculos entre el mundo, el estudiante y cómo desarrolla formas de pensar. Lo interesante del texto es que trata de establecer los vínculos desde la pedagogía crítica para entender las nuevas realidades que enfrenta la educación superior. Señala que en la cotidianidad es donde se observa a las personas y estas pueden encontrar los caminos para entender qué pasa. Los profesores deben pensar sus prácticas con relación al lugar y las personas con las que interactúan. 
Desde una perspectiva diferente, Díaz (2012) observa la cotidianidad de los estudiantes; para ello, sale de las aulas en busca del centro de la vida universitaria y encuentra que lo más importante está en la cotidianidad, en los espacios donde los estudiantes comparten. Los espacios en el campus donde los estudiantes se sienten bien son diferentes a las aulas de clase; son los espacios que ellos construyen o de los cuales se apropian.

Incluso, Blanco (2014a, 2014b) estableció que lugares como los baños y las fiestas eran importantes para estudiar la sexualidad en los estudiantes. Los baños son espacios de indicaciones de intercambios para estudiantes que pertenecen a grupos de distintas sexualidades. Las fiestas se proponen como un evento institucional donde el modelo heterosexual se presenta como normal. En ambos espacios los estudiantes representan o intercambian contenidos sexuales. La universidad se convierte en un espacio para establecer relaciones, regulaciones de género y sexualidades (Blanco, 2014a). La investigación se orientó a la vida cotidiana, sentidos de las expresiones de género y sexualidad en los espacios. La universidad es un espacio de manifestación y discusión de diferencias, donde se plasman las subjetividades presentes, de forma subterránea y en las representaciones institucionales.

Rodríguez (2011) hizo una etnografía que relacionó la vida de los jóvenes, la dinámica de la universidad y las particularidades de la ciudad. Las instituciones donde se desarrolló la investigación eran públicas; sin embargo, se observaron barreras entre los estudiantes, separados por nosotros y ellos. Se evidenciaron barreras entre estudiantes que no tenían diferencias económicas, sociales y culturales significativas. Indagando sobre el motivo de la desigualdad entre ellos, Rodríguez encontró que la diferencia radicaba en los lugares de procedencia y los espacios donde concurrían cotidianamente; esto creaba subjetividades que identificaban a ciertos grupos con determinados lugares. Las barreras se referían a espacios de la ciudad; a pesar de ser dimensiones diferentes, los lugares de procedencia afectaban los procesos educativos y las relaciones entre los estudiantes.

Soler et al. (2011) toman la participación de los estudiantes en distintos ámbitos del funcionamiento de la universidad, establecen vínculos con sus experiencias y la cotidianidad institucional. El estudio fue motivado por la poca participación de los estudiantes en los órganos de decisión de la universidad. Pese a las orientaciones de organismos de dirección, incluso internacionales, los resultados de la investigación presentaron un evidente desinterés por parte de los estudiantes en cuanto a participar en instancias administrativas o políticas de la institución (Soler et al., 2011).

Para agregar un elemento a la experiencia estudiantil, Zibecchi (2002) estudió a los universitarios en un contexto de la crisis económica y social. Los estudiantes analizados manifiestan que en su cotidianidad vivieron situaciones de desesperanza y de incertidumbre. La propuesta de Zibecchi 
fue dar cuenta de las transformaciones estructurales desde las experiencias y las percepciones de los estudiantes. En su estudio encontró que había una creciente incertidumbre por parte de los alumnos sobre cómo sería su futuro una vez se recibieran como profesionales, debido a la inestabilidad económica, las crisis sociales y la precariedad laboral.

Los espacios universitarios marcan las experiencias estudiantiles, se convierten en posibilidades para las personas que participan en ellos. Los campus se vuelven medios donde los estudiantes desarrollan sus potencialidades. Los territorios son conquistados o construidos por ellos, es su tarea apropiarse de lugares que se convierten en el centro de sus vidas. Por último, se presenta la universidad como un espacio alternativo frente a las crisis sociales que surgen en diferentes momentos; allí los estudiantes pueden observar con herramientas fundamentadas la situación en que se encuentran para buscar alternativas. Entonces, el espacio supera lo académico para convertirse en un refugio o una construcción de los estudiantes.

\section{Los vínculos: intercambios y grupos diversos}

Algunas investigaciones muestran la experiencia estudiantil a partir de los vínculos que se tejen durante su proceso de formación, relacionados directamente con las particularidades de los individuos frente a los estereotipos presentes en la educación superior. Si bien los lazos suelen ser académicos, es posible que exista otro tipo de conexiones alejadas de lo educativo. Se destacan las investigaciones sobre los vínculos de los estudiantes en los intercambios que se dan cuando las personas se trasladan para continuar con sus estudios universitarios (Rufatt, 2011; Subuhana, 2009; Yubero y Larrañaga, 2011) y los grupos diversos que crean espacios en la universidad y se enfrentan con los estereotipos imperantes (Blanco, 2014b; Chávez, 2008; Cureton, 2003; Grant, 1997; Quintero, 2014).

Se presenta un fenómeno cada vez más usual: los intercambios de jóvenes que abandonan su lugar de origen y viven en un nuevo sitio, sin olvidar el viejo y sin adaptarse al nuevo (Dubet, 2010), lo que crea una creciente migración de los estudiantes universitarios. La movilidad es recurrente en diferentes contextos. Aquí, se presentan tres visiones del fenómeno: migración de provincia a la capital, la intercontinental y, una particular, cómo los locales reciben a los migrantes.

Rufatt (2011) analiza los procesos de adaptación de jóvenes que migran de provincia a la capital para estudiar en una universidad importante. Los jóvenes que llegan allí se encuentran con el frío de la gran ciudad y de la universidad, en contraste con la calidez de su comunidad de origen. Los jóvenes son conscientes de que en provincia tienen pocas oportunidades mientras que en la capital se abre un abanico laboral y 
profesional que los motiva a soportar esta situación. Los participantes destacan las dos caras de la migración: en provincia tenían mejor calidad de vida, ritmos y vínculos fraternales con familia y amigos; en la capital, logros profesionales, recursos intelectuales, campo laboral amplio y ascenso en el estatus social.

Una experiencia particular de migración es la documentada por Subuhana (2009) sobre la movilización de estudiantes universitarios de África Lusófona a São Paulo, Brasil. Se indaga acerca de su llegada, su adaptación, sus descubrimientos y sus perspectivas de retorno. El viaje los hace alejarse de sus dinámicas sociales y culturales de origen, para adoptar pautas que una ciudad como São Paulo les ofrece; allí encuentran un nuevo modo de vida. La migración de los africanos continúa: la primera es del campo a la ciudad, luego de su país a otro; puede considerarse que buscan condiciones para una vida mejor, generalmente, lejos del país de origen. La formación universitaria es el camino para mejorar su calidad de vida.

Desde una perspectiva diferente, Yubero y Larrañaga (2011) analizan la imagen social que tienen los estudiantes universitarios españoles vinculados al campo educativo sobre los inmigrantes. La elección de este grupo corresponde a que en su futuro profesional se verán obligados a mantener relaciones con niños y jóvenes migrantes provenientes de países del "tercer mundo" (en su mayoría de Centro y Sur América), que llegan a Europa. Allí, representaciones creadas por informes e interpretaciones de personas o instituciones $y$, sobre todo, por noticias o imágenes en los medios de comunicación, van creando reacciones y barreras entre los locales y los migrantes. Muchas en la cotidianidad están cargadas de un "racismo simbólico", que genera una valoración de los grupos originarios entre la expulsión o el intercambio.

La migración de los estudiantes es un fenómeno complejo. Por lo general, en las universidades encuentran espacios que posibilitan su permanencia y su formación; fuera de ellas, se encuentran con los problemas comunes que afrontan los migrantes de países del "tercer mundo" que llegan al "primer mundo".

El segundo tema son los grupos diversos que representan a los estudiantes. El primero que se destaca es el racismo en la universidad. Cureton (2003) indagó sobre la experiencia de estudiantes afrodescendientes en un contexto tradicional y predominantemente blanco, donde podrían verse afectados en el desarrollo de sus actividades académicas por manifestaciones de hostilidad. Desde la experiencia de los estudiantes negros se concluyó que no vivieron o notaron discriminación o referencias racistas.

Desde otra perspectiva, Grant (1997) demostró la marginación de los afroamericanos en un campus de Estado Unidos de América. En su estudio, analizó cómo los estudiantes enfrentan su condición de marginales, 
construyen significados y asumen sus experiencias. Son parte de un grupo con una larga tradición de marginalidad, que ha desarrollado estrategias para enfrentarla. Del mismo modo, Quintero (2014) aborda el racismo y la discriminación en la educación superior y, de paso, en la sociedad. Se centra en las experiencias de los estudiantes afrocolombianos en Bogotá, que históricamente han sido objeto de discriminación, ya que las dinámicas y los principios de la universidad en Colombia han reproducido el racismo e invisibilizado sus acciones. Quintero demostró que existen prácticas de racismo latentes, incrustadas en el funcionamiento y la operatividad de las universidades, donde las distancias están determinadas y lo que se debe hacer es mantenerlas, el racismo forma parte de la cotidianidad de las universidades (2014).

La participación de los estudiantes en la vida universitaria depende de la situación social o el lugar de desempeño en la universidad. Chávez (2008) observa la cotidianidad, centrado en mecanismos, prácticas y estrategias que los estudiantes indígenas desarrollan a su paso por estas instituciones. En América Latina, existen diferentes manifestaciones de discriminación dentro de las universidades. Chávez (2008) indaga sobre cómo los estudiantes indígenas enfrentan una situación social y académica adversa. Se destacan los problemas académicos y las relaciones personales, donde constantemente se manifiesta su condición de pertenencia a grupos distintos. En el caso indígena, se utilizan etiquetas o estigmas, adjetivos que atacan su condición o su grupo. Se critica su forma de vestir, de actuar, de hablar, de pensar diferente, que riñe con las personas que son producto un sistema educativo homogéneo y donde poco se acepta lo heterogéneo. Frente a este panorama, los estudiantes desarrollan formas de adaptación institucional a través redes de amistad con personas cercanas y apoyándose en programas institucionales. Para enfrentar la adversidad, constantemente están reivindicando y reconociendo sus orígenes y sus particularidades como comunidad, para fortalecer la autoestima en los jóvenes y afrontar un proceso hostil.

Por otro lado, Blanco (2014b) indaga sobre la diversidad sexual. Son demandas y reivindicaciones de movimientos feministas o de diversidad que conforman comunidades universitarias. Se propone que participar en procesos de identificación colectiva deja huella en las personas. Desde luego, encuentran obstáculos en el camino para la construcción de su comunidad; los estereotipos tradicionales están presentes en la cotidianidad universitaria, a pesar de que se alejan de la condición actual de los jóvenes.

Los vínculos que se tejen en los intercambios o en la participación en grupos que son cercanos, influyen de forma significativa en la construcción de la experiencia estudiantil. El hecho de que las personas deban moverse y establecer intercambios en su nueva condición crea dificultades en su adaptación y de rendimiento académico; por esta razón, deben desarrollar 
estrategias para continuar su formación en ambientes nuevos para ellos. De igual manera, en muchos espacios se utilizan etiquetas y acciones para discriminar a personas diferentes, por el lugar de procedencia o por ser miembros de un grupo social; lo importante de esta observación es cómo las personas frente a la adversidad buscan opciones que les permitan terminar su proceso de formación. La participación en intercambios o grupos diversos son algunos de los vínculos que se desarrollan en la experiencia estudiantil.

\section{A manera de cierre}

Los referentes básicos establecen unas condiciones para el estudio sociológico de la experiencia estudiantil en la universidad. Parten de la exigencia de indagar sobre los protagonistas del proceso de formación; la necesidad de escucharlos, de compartir, de entender qué sucede con las personas que participan en la formación. Las actividades son: 1) estudiar, que continúa siendo la acción más importante pero ha sido desplazada por otras; 2) trabajar, lo que permite a los estudiantes obtener inicialmente los recursos para continuar su formación y encontrar un campo dónde desarrollarse laboralmente; y 3) militar, como una actividad en la que buscan ser parte de un grupo y manifestar sus puntos de vista frente a situaciones o vivencias que les conciernen o los afectan. Luego, se abordan los espacios, diferentes a las aulas, que los estudiantes comparten o crean; son construcciones donde participan y en algunos casos se convierten en el centro de su formación. Durante su paso por la universidad los estudiantes construyen vínculos a partir de intercambios o de su participación en grupos diversos, el proceso va desde la adaptación en un ambiente de cordialidad hasta problemas que pueden convertir esta experiencia en una pesadilla para algunos. Los referentes básicos, las actividades destacadas, los espacios y los vínculos son los temas que sintetizan los tratamientos que se dan a la experiencia estudiantil.

Sin embargo, las investigaciones sobre este tema, por lo general, dejan al margen el entorno en el que se desenvuelven las personas. La producción derivada de los modelos sociológicos tradicionales parte de un orden social, del equilibrio de poderes donde el Estado regula las relaciones entre ciudadanos y establece los monopolios de la violencia y los tributos (Elías, 1997). Según estas perspectivas, cuando el orden social sufre alteraciones y cambia la dinámica, se genera un conflicto social y la intervención del Estado se centra en ponerle fin y volver a regular las relaciones entre ciudadanos. Esta visión se deriva de la sociología clásica, que tomó como modelos ideales a Francia, Inglaterra y Estados Unidos (Martucelli, 2012) y estableció distancias con sociedades que no correspondían con su propia teoría creando términos como "civilización o barbarie", "desarrollo o 
subdesarrollo". Sin embargo, el problema era que las sociedades respondían a otras lógicas, no eran buenas o malas, solo diferentes. Así, es difícil que este tipo de explicaciones satisfagan la necesidad de entendimiento en sociedades diferentes.

Por tal motivo, se abre la necesidad de encontrar herramientas que permitan entender la experiencia estudiantil en sociedades diferentes, como América Latina. Sociedades donde, en ciertos casos, el Estado no regula las relaciones entre ciudadanos sino que crea barreras profundas entre grupos que participan. Dependiendo del lugar, no existen monopolios de la violencia, en ocasiones el Estado es quien viola los derechos de las personas y los tributos son parte de los recursos de los grupos que dominan el Estado.

En estos contextos, el análisis de la experiencia estudiantil cambia. Siguiendo la tradición de estudios en sociología de la educación, el aprendizaje y la vinculación con las instituciones ocupan lugares importantes, pero no son todo. En estos contextos, las personas toman rutas alternativas para desarrollar su proceso de formación, buscando recursos que les permitan continuar y herramientas para entender y modificar su condición individual en un contexto adverso. En esta búsqueda, las personas sufren una serie de transformaciones que se evidencian en la experiencia estudiantil. Son pocas las investigaciones que establecen una relación significativa entre la experiencia estudiantil y la sociedad donde se desarrolla, los vínculos entre la formación y los problemas sociales que afectan a los estudiantes quedan al margen, para continuar limitando al estudiante a su oficio de estudiar y confinándolo a un espacio como la universidad, sin tener en cuenta otros espacios donde desarrolla parte de su vida y que definen su experiencia.

\section{Referencias}

Acosta, F., Cubides, J. y Galindo, L. (2011). Sentidos y prácticas políticas en el mundo juvenil universitario. Bogotá: Universidad Nacional de Colombia.

Amaya, R., Gómez, M. y Otero, A. (2007). Autonomía universitaria y derecho a la educación: alcances y límites en los procesos disciplinarios de las instituciones de educación superior. Revista de Estudios Sociales, 26, 158-165. Recuperado de http://dialnet.unirioja.es/servlet/articulo?codigo $=2345381$.

Arango, L. (2006). Jóvenes en la universidad. Género, clase e identidad profesional. Bogotá: Universidad Nacional de Colombia. 
Archila, M. (2012). El movimiento estudiantil en Colombia, una mirada histórica. Revista del Observatorio Social de América Latina, 31(13), 71-104. Recuperado de http://bibliotecavirtual.clacso.org.ar/clacso/ osal/20120417105250/OSAL31.pdf.

Barnes, N. (1992). The fabric of a student's life and thought: Practicing cultural anthropology in the classroom. Anthropology \& Education Quarterly, 23(2), 145-159. Recuperado de: http://www.jstor.org/stable/3195952.

Barragán, D. (2017). La experiencia estudiantil en una sociedad hostil. Los estudiantes universitarios de Ibagué (2012) (tesis doctoral). Universidad de Buenos Aires, Buenos Aires, Argentina.

Benavides, A., Francischetto, V., Marqués, B., Miranda, J., Nogueira, C., Leme, V., Araújo, A. y Almeida, L. (2014). O impacto das expectativas na adaptação acadêmica dos estudantes no Ensino Superior. Psico-USF, 19(1), 49-60.

Blanco, R. (2014a). Normatividades de la vida cotidiana. Género y sexualidad en los saberes, la política y la sociabilidad universitaria. Propuesta Educativa, 42, 55-64.

Blanco, R. (2014b). Intimidades públicas: experiencia estudiantil y normatividad sexo-genérica en las instituciones universitarias. Intersticios, $8(1), 157-170$.

Blanco, R. (2014c). Estudiantes, militantes, activistas. Nuevas agendas de las agrupaciones universitarias en torno al género y la diversidad sexual. Perfiles Educativos, 35(144), 140-156.

Bonavena, P. y Millán, M. (2012). El movimiento estudiantil en la actualidad argentina: una aproximación sociohistórica. Revista del Observatorio Social de América Latina, 31(13), 105-122. Recuperado de http://bibliotecavirtual.clacso.org.ar/clacso/osal/20120417105250/ OSAL31.pdf

Bourdieu, P. (2004). El baile de los solteros. La crisis de una sociedad campesina en Bearne. Barcelona: Anagrama.

Camou, A., Prati, M. y Varela, S. (2014). Tras las huellas de la participación política. Un estudio sobre la experiencia reciente de estudiantes universitarios. Universidades, 45(60), 6-25.

Carli, S. (2012). El estudiante universitario. Hacia una historia del presente de la educación pública. Buenos Aires: Siglo xxı.

Carrasco, G. (2010). Participación y tendencias políticas en estudiantes universitarios: el caso de la Universidad de Chile. Última Década, 32, 85-103. Recuperado de http://www.scielo.cl/scielo.php?pi$\mathrm{d}=$ S0718-22362010000100005\&script=sci_arttext. 
Casillas, M., De Garay, A., Vergara, J. y Puebla, M. (2001). Los estudiantes de la UAM-A, un sujeto social complejo. Revista Mexicana de Investigación Educativa, 6(11), 1-16.

Chávez, M. (2008). Ser indígena en la educación superior ¿desventajas reales o asignadas? Revista de la Educación Superior, 37(148), 31-55.

Cubides, H. y Guerrero, P. (2012). Trazos e itinerarios políticos de jóvenes. Bogotá: Universidad Central.

Cureton, S. (2003). Race-specific college student experiences on a predominantly white campus. Journal of Black Studies, 33(3), 295-311.

Díaz, A. (2012). Cotidianidad, cultura y territorio en la vida del estudiantado universitario (tesis doctoral). Universidad de Málaga, Málaga, España. Recuperado de http://riuma.uma.es/xmlui/handle/10630/4982.

Donoso, S. (2014). La reconstrucción de la acción colectiva en Chile postransición: el caso del movimiento estudiantil. Reporte. Centro de Estudios de Conflicto y Cohesión Social (COEs). Septiembre. Recuperado de http://biblioteca.clacso.edu.ar/clacso/becas/20140905014946/ Donoso_Informe_Sep_2014.pdf.

Dubet, F. (2005). Los estudiantes. Revista de Investigación Educativa, 1, 2-78. Recuperado de http://www.uv.mx/cpue/num1/inves/completos/Dubet.pdf.

Dubet, F. (2010). Sociología de la experiencia. Madrid, España: Editorial Complutense-CIS.

Dubet, F. (2011). La experiencia sociológica. Barcelona: Gedisa.

Dubet, F. y Martucelli, D. (1998). En la escuela. Sociología de la experiencia escolar. Buenos Aires: Lozada.

Duschatzky, S. (2008). La escuela como frontera. Reflexiones sobre la experiencia escolar de jóvenes de sectores populares. Buenos Aires: Paidós.

Elías, N. (1990). Compromiso y distanciamiento ensayos de sociología del conocimiento. Barcelona: Península.

Elías, N. (1997). El Proceso de la civilización. Investigaciones sociogenéticas y psicogenéticas. Bogotá: FCE.

French, A. (1997). The experience of college undergraduates: Degrees of transformation (tesis de doctorado). Cranfield, Cranfield University, Reino Unido. Recuperado de https://dspace.lib.cranfield.ac.uk/handle/1826/3376.

Gómez, M. y Alzate, M. (2010). El "oficio" de estudiante universitario: afiliación, aprendizaje y masificación de la Universidad. Pedagogía y Saberes, 33, 85-97.

Graff, G. (1995). Conflict pedagogy and student experience. College Composition and Communication, 46(2), 276-279. Recuperado de http://www.jstor.org/stable/358431. 
Grant, B. (1997). Disciplining students: The construction of student subjectivities. British Journal of Sociology of Education, 18(1), 101-114.

Guevara, H. (2009). Identidades estudiantiles, conocimiento y cultura. Percepciones de jóvenes universitarios y universitarias de Cuyo, Argentina. Revista Latinoamericana de Ciencias Sociales, Niñez y Juventud, 7(1), 209-234.

Guzmán, C. (2002). Reflexiones en torno a la condición estudiantil en los noventa: Ios aportes de la sociología francesa. Perfiles Educativos, 25(98), 38-56.

Kaplan, C. (2008). Talentos, dones e inteligencias: el fracaso escolar no es un destino. Buenos Aires: Colihue.

Kessler, G. (2002). La experiencia escolar fragmentada. Estudiantes y docentes en la Escuela Media de Buenos Aires. Buenos Aires: IIPE-Unesco.

Lahire, B. (2006). El espíritu sociológico. Buenos Aires: Manantial.

Larotonda, C. (2007). Las movilizaciones estudiantiles de 2006: una respuesta a mitos y esperanzas defraudados. Polis, Revista de la Universidad Bolivariana, 16(5), 1-29. Recuperado de http://www.redalyc. org/articulo.oa? $\mathrm{id}=30501615$.

Lozano, M. (2009). La política, la democracia y la ciudadanía en los juicios, discursos y acción política en grupos de jóvenes estudiantes universitarios de Bogotá (tesis doctoral).Universidad de Manizales, Manizales, Colombia.

Lozano, M. y Alvarado, S. (2011). Juicios, discursos y acción política en grupos de jóvenes estudiantes universitarios de Bogotá. Revista Latinoamericana de Ciencias Sociales, Niñez y Juventud, 9, 101-113. Recuperado de http://revistaumanizales.cinde.org.co/index.php/Revista-Latinoamericana/article/view/346/209.

Malinowski, N. (2008). Diferenciación de los tiempos estudiantiles e impacto sobre el proceso de afiliación en México. Revista Latinoamericana de Ciencias Sociales, Niñez y Juventud, 6(2), 801-819.

Martuccelli, D. (2012). Las sociologías del individuo. Santiago: ıom Ediciones

Mira, A. (2011). Crisis de representatividad y estallido social. Una aproximación a la actual experiencia chilena. Polis, 10, 185-197. Recuperado de http://www.scielo.cl/scielo.php?script=sci_arttext\&pid=S0718-65682011000300009\&nrm=iso.

Murcia, N. (2006). Vida universitaria: un estudio desde los imaginarios de maestros y alumnos (tesis doctoral). Universidad de Manizales, Manizales, Colombia. Recuperado de http://biblioteca.clacso.edu. ar/Colombia/alianza-cinde-umz/20100201034418/TESISNAPOLEONMURCIA.pdf.

Nobile, M. y Arroyo, M. (2015). Los efectos de experiencias escolarizadoras inclusivas sobre los relatos biográficos de docentes y estudiantes: 
un análisis de las Escuelas de Reingreso en Ciudad de Buenos Aires. RASE. Revista de la Asociación de Sociología de la Educación, 8(3), 409-424.

Ouviña, H. (2012). Somos la generación que perdió el miedo. Entrevista a Camila Vallejo Dowling. Revista del Observatorio Social de América Latina, 31(13), 13-22. Recuperado de http://bibliotecavirtual.clacso. org.ar/clacso/osal/20120417105250/osal31.pdf.

Paoloni, P. (2011). Trayectorias laborales y académicas. Puntos de encuentro y de desencuentro en estudiantes universitarios. Revista Iberoamericana de Educación, 55(2), 1-11. Recuperado de http://www.rieoei. org/deloslectores/3283Paoloni.pdf.

Picotto, D. y Vommaro, P. (2010). Jóvenes y política: las agrupaciones estudiantiles independientes de la Universidad de Buenos Aires. Nómadas, 32, 149-161.

Pinzón, C., Garay, G. y Suárez, R. (2008). Para cartografiar la diversidad del@s jóvenes. Bogotá: Universidad Nacional de Colombia.

Quintero, O. (2014). El racismo cotidiano en la universidad colombiana desde la experiencia vivida por los estudiantes negros en Bogotá. Universitas Humanística, 77, 71-94. Recuperado de http://www.redalyc.org/pdf/791/79130107004.pdf.

Rockwell, E. (1997). De huellas, bardas y veredas. Una historia cotidiana en la escuela. En La escuela cotidiana. México: FCE.

Rodríguez, M. (2011). Mundos urbanos: el contacto con el "otro" y la producción de la diferencia en la ciudad (tesis doctoral). Universitat Rovira i Virgili. Recuperado de http://tesisenred.net/handle/10803/37345.

Rojas, M. (2009). El abandono de los estudios: deserción y decepción de la juventud. Holograma, 10(4), 75-94. Recuperado de www.alfaguia.org/alfaguia/files/1319736384_13.pdf.

Roldán, O. (2014). Incursión de los y las jóvenes en el mundo universitario: tensiones entre ser y permanecer. Educação \& Sociedade, 35(126), 143-160. Recuperado de https://dx.doi.org/10.1590/S010173302014000100009.

Ruesga, S., Silva, J. y Monsueto, S. (2014). Estudiantes universitarios, experiencia laboral y desempeño académico en España. Revista de Educación, 352, 67-95. Recuperado de http://www.mecd.gob. es/dctm/revista-de-educacion/articulos346/05I.estudiantesuniversitriosrev.ed.365.pdf?documentld=0901e72b819f0eb6

Rufatt, M. (2011). Calidad de vida en migrantes universitarios asentados en la región metropolitana de Santiago de Chile. Hologramatica, 7(14), 11-40. Recuperado de http://www.cienciared.com.ar/ra/usr/3/1091/ hologramatica_n14_v1pp37_40.pdf. 
Sandoval, J. y Hatibovic, F. (2010). Socialización política y juventud: el caso de las trayectorias ciudadanas de los estudiantes universitarios de la región de Valparaíso. Última Década, 32, 11-36. Recuperado de: http://www.scielo.cl/pdf/udecada/v18n32/art02.pdf.

Soler, P., Vilá, M., Fullana, J., Planas, A. y Pallisera, M. (2011). La opinión de los estudiantes sobre su participación en la universidad. Educatio Siglo XxI, 29(1), 323-344. Recuperado de http://revistas.um.es/educatio/article/view/119991.

Subuhana, C. (2009). A experiência sociocultural de universitários da África Lusófona no Brasil: entremeando histórias. Pro-Posições, 20(4), 103126. Recuperado de http://www.scielo.br/pdf/pp/v20n1/v20n1a07.

Vázquez, L. (2009). ¿Estudias y trabajas? Los estudiantes trabajadores de la Universidad Autónoma Metropolitana. Revista Latinoamericana de Estudios Educativos, 39(3-4), 121-149. Recuperado de http://www. redalyc.org/pdf/270/27015078006.pdf.

Weiss, F., Klein, M. y Gravenhorst, R. (2014). The effects of work experience during higher education on labor market entry: Learning by doing or an entry ticket? Work, Employment \& Society, 28(5), 788-807.

Yair, G. (2008). Key educational experiences and self-discovery in higher education. Teaching and Teacher Education, 24, 92-103.

Yubero, S. y Larrañaga, E. (2011). La influencia educativa en la construcción de la imagen social de los inmigrantes en estudiantes universitarios. Boletín Informativo de Trabajo Social-BıTs, 16. Recuperado de http://www. uclm.es/bits/sumario/09.asp.

Zibecchi, C. (2002). Universidades argentinas en un contexto neoliberal: un análisis centrado en la experiencia y las percepciones de sus estudiantes. Documento de trabajo. Buenos Aires: Consejo Latinoamericano de Ciencias Sociales-Clacso. 